\title{
Agent-Based Modelling as a Tool for Testing Electric Power Market Designs
}

\author{
Marijn Maenhoudt \\ Department of Electrical Engineering \\ Katholieke Universiteit Leuven \\ marijn.maenhoudt@esat.kuleuven.be
}

\author{
Geert Deconinck \\ Department of Electrical Engineering \\ Katholieke Universiteit Leuven \\ geert.deconinck@esat.kuleuven.be
}

\begin{abstract}
Although agent-based modelling history can be traced back until the 1960's in computer sciences, the technology still slowly trickles down from theoretical abstraction to practical application. Interesting study areas have been using agent-based models to assess their research questions for a long time while other fields of study which are interesting from an agent-based technology perspective - such as management sciences - only recently recognised this technology. This paper sets an overview on the benefits and criticism of agent-based models and shows how this tool can be employed to gain better insights in complex market designs, policies and principles with respect to the more traditional tools. Especially in the field of wholesale electric power markets, agent-based models gleam as a promising market design deliberation tool anterior to the actual real world market implementation. An introduction to the latest electricity market models is given to illustrate how national and international policy makers embrace agent-based simulations as a framework to base their decisions upon.
\end{abstract}

Index Terms-Multi-agent system, agent-based, simulation, modelling, electric power market, Optimate

\section{INTRODUCTION}

A 11 existing electric power markets have evolved significantly from the moment the market became accessible for competition. Boldly stated, this evolution was a consequence of the failure of initial electricity market designs to grasp the whole complexity of a free electricity market. In the US, the standard market design proposal (SMD) in July 2002 failed due to political, regional and stakeholder pressures and was therefore adapted by the less ambitious Wholesale Power Market (WPM) proposal [1]. The costs of implementing the SMD proposal were epitomised by the Northeast Blackout of 2003 and the Californian electricity market debacle [2]. Also in Europe electricity market structures were forced to adapt after bearing heavy criticism. David Newbery describes in his work why the UK decided to supersede the Pool market concept by the New Electricity Trading Arrangements (NETA) framework and which other difficulties arose after doing so [3]. Norway's electricity market reform failure is described in the work of Chi-Keung Woo and Debra Lloyd [4]. In general, the market reform failures are attributed to transmission congestion abuses by few dominant sellers, poor market designs that invites strategic bidding by suppliers, the lack of customer response

This project is supported by the European Commission in the Seventh Framework Programme to price spikes, capacity shortage caused by demand growth not matched by new capacity and thin trading of forward and futures contracts that are critical for price discovery and risk management. The evolution of electricity markets thus signifies the search towards reliable services and stable prices. Evolution is an indispensable demonstration that one learns from its mistakes, especially in complex matters. With the implementation of such a huge reform as the liberalisation of the electricity market, one first has to gain experience in order to optimise an initial conceptual model. After all economic, social and environmental factors characterise the electricity markets and need to be included in the market design in order to obtain an undivided policy. Traditional economic policy analysis models such as Computable General Equilibrium models (CGE) are unable to reproduce complex behaviours and only represents the economic side of the system, leading to an incomplete representation of the system and an incomplete policy decision. Other economic measures for market efficiency such as the Herfindahl-Hirschman Index also do not cover all important factors that determine efficient market operation.

Yet there exists a tool to incorporate more than economical interactions in the analysis: agent-based modelling. Following this introductory section, the second section of this paper gives an introduction to the concept of agent-based simulation. A summary of the discussion about the strengths and weaknesses of agent-based modelling is presented followed by applications where agent-based simulation is already accepted as a policy or market assessment tool. The third section focuses only on the electricity power market application domain. It takes a closer look on how agent-based modelling has been used recently to simulate the complex behaviour of market participants in the electricity markets in order to gain better insights on how market power is exacerbated by market participants. Furthermore three important agent-based simulation frameworks - EMCAS, AMES and OPTIMATE - that attempt to model the strategic trade of electricity power are discussed. As conclusion the fourth section of this paper summarises the potential of agent-based modelling to simulate electricity market designs and highlights which steps are needed to improve the existing models of today. 


\section{Agent-Based Simulation: A more Complete MODELLING TOOL}

Providing a complete and precise definition of agents is the subject of many papers in computer sciences. The weak notion proposed by Wooldridge and Jennings to describe agents as entities having the ability to react, pro-act and interact can lean on a broad consensus but this definition is the subject of much discussion among computer scientists as this notion sums up general features instead of giving an exact definition [5] [6]. Without depicting this discussion any further one can generalise in the context and scope of this paper that individual agents are capable of autonomously engaging in high-level interactions within an organisational relationship in order to obtain their own goals and objectives through the maximisation of a utility function. An agent then tries to fine tune its utility function maximisation by learning how actions can be exerted for its own gains.

\section{A. Benefits and Advantages}

A first strength of agent-based modelling comprises of the attributes of the individual agents. Weiss emphasises the strong notion of autonomy and the social behaviour between agents while Wooldridge portrays agents as highly disciplinary [7]. Based on an agent's own perceptions of the environment and the implications of actions of other agents on the environment an agent decides for itself whether or not to act or to interact. This distinctive logic and social behaviour makes agent-based modelling an interesting tool for research disciplines containing strategy and physics, ranging from economy to ecology and philosophy [8].

A second strength involves so-called emergent behaviour. By modelling innumerable elementary micro-scale interactions between agents - each having their own objectives, their own characteristics and their own ability to make decisions complex macro-scale aggregate dynamics emerge. Multi-agent systems will thus be constructed to model systems characterised by complex interactions involving multiple parties such as traffic problems, social behaviour, human decision making and market mechanisms. The theory of emergent behaviour allows for less abstraction from the details of a phenomenon, even when simulated reality is poorly understood. From this point of view no ideal-case assumptions are formulated on beforehand, thereby eliminating their potential implications in the observed system, so that even unexpected aggregated behaviour and new insights can arise from the simulation.

Thirdly, but affiliated with the second advantage, agent-based modelling reduces system control complexity by replacing the aggregated outcome of a phenomenon, which is most of the time difficult to explain or to predict, with uncomplicated decision rules of self-aware entities at a lower level. Decentralising complex systems is a natural representation of reality as it abates the burden of a high degree of coupling between individual entities. Agents themselves make decisions, establish interactions, deal with requests and spontaneously generate requests whenever the taken measures are aligned with their overall intentions at run-time [9].
A fourth strength covers the ability to allow multi-step simulation in order to observe events as they unfold over time. The top level goals need not to be defined but is rather a consequence of the accomplishment of each agent objectives. This feature of agent-based modelling allows testing of diverse scenarios and distinct agent strategies by modifying simulation parameters [10].

\section{B. Criticism and Objections}

Besides the auspicious benefits of agent-based simulation, criticism is likewise present in academic circles with an equivalent loud voice. A first argument concerns the qualitative nature of agent-based simulation. Quantitative data in terms of efficiency, reliability or productivity with respect to other technologies is lacking. The prime challenge to overcome this criticism is for agent-based models to provide new insights into complex systems that cannot be provided by the traditional approaches. But how can one use agent-based modelling when it is strenuous to validate the performance of the model against empirical data [10]?

A second argument emphasises the reliability issue of agentbased simulation. Take for example the emergence of unexpected aggregated behaviour which is cited by supporters as strength of agent-based technology for leading to new hindsight. Equally well this emergent behaviour is triggered by a flaw in the simulation topology. Once again the difficulty of validating the performance of an agent-based model itself against empirical data is the impediment to be surmounted in order to clear away this point of criticism [11].

Validating agent-based models is indeed an intricate part as the model has to withstand criticism and scrutiny from the many involving stakeholders - the agents in the model - each having their own bias and interests in what the model results may imply. Traditional model validation comprises systematic comparison of the simulation model results to real world data using statistical tools. This is however not an option when using complex agent-based models. Macal states that simulation result comparison with the results of a participatory simulation - where real persons preferably laymen on the matter take up the role of agents - already serves as a useful validation tool [12]. Although this approach gains insights on which parameters are focused upon by agents to cultivate a strategy, this author beliefs that intensive communication throughout the development phase of the model towards all participants and decision makers is a more solid approach to eliminate credibility barriers. Agent-based modelling leans on decentralising complex systems, so that intuitively the validation should also be focused upon solidifying the simpler decision rules on the agent level. This approach seems more feasible than a validation of the aggregate model, especially with large-scale models consisting of hundreds or thousands of agents.

A second weakness of agent-based modelling concerns the flexibility of agent interactions. Agents decide themselves whether to grant a request or to refuse it at a certain moment in time based on the objective the agent finds most important 
to pursue at that time. When running the same agent-based model another time, the agent may find another objective more important and will thus exert other actions. The patterns and effects of agent interactions are thus uncertain and change when rerunning the simulation even with the same simulation parameters. This flexibility leads to unpredictability during run-time, especially for systems with hundreds to thousands of agents. The interpretation of the results is thus not to predict the future but to provide insights into the probability of possible evolutions of the system.

\section{Applications of Agent-Based Models}

Parunak lists key study area characteristics for recognising applications of agent-based models. The aggregate outcome of the system should result from a multitude of complex micro-scale interactions between innumerable agents where each agent makes its own unique decisions and has its own learning process in order to adapt its goal-achieving strategy to the changing environment. The system is thus characterised by many interrelated factors, high uncertainty, many complex interactions and adaptive behaviour. The agentbased simulation tools are subsequently tailored for analysing, planning, testing and validating economic and social concepts, hypothesis or policies as will be seen in the next examples [13]. Balmann developed the AgriPoliS model which has been used by Happe to evaluate the impact of the EU Agenda 2000 and other proposed agricultural policies on regional structural change in the Hohenlohe region, Germany [14] [15]. The ALMaSS model was created to evaluate the impact of human management of landscapes on animal species in Denmark. Moving from the ecological policy perspective to the political economics, the US Department of Justice complements structural analysis with agent-based simulation to analyse mergers in differentiated product industries [16].

Logistics problems in the area of traffic management have been studied by Wolfe who evaluated strategies for the airline operations centre agents to select routes through collaborative air traffic flow management by order of the NASA [17]. STREETS on the other hand is an agent-based pedestrian model used for investigating pedestrian behaviour in urban centres [18].

Implementation of advanced agent-base Enterprise Resource Planning (ERP) systems in the manufacturing industry increase supply chain throughput and make the supply chain more robust and responsive to prevailing circumstances [19]. A more strategic example studies the dynamics and outcomes of temporal price competition [20].

One can deduct from the illustrations above that the scope of agent-based modelling gradually broadens from a theoretical concept in computer science towards a helpful software tool for multiple research domains. The continuation of the paper takes a closer look on another challenging application of agent-based modelling, the research domain of electric power markets.

\section{Agent-Based Modelling of Electric Power MARKETS}

As indicated in the introduction the economic aspect is not the sole factor which determines the electricity market outcome. The social and environmental factors originating from the many market players and technical constraints involved cannot be neglected. The subsequent modelling of the highly complex and dynamic characteristic of the electric market system is a challenge which can be taken up by agent-based simulation tools. This section gives a presentation of both existing and future models.

\section{A. The EMCAS model}

The Electricity Market Complex Adaptive System (EMCAS) model is an electricity market model designed to investigate market restructuring of the power market and to understand implications of a competitive market on electricity prices, availability and reliability. The research question EMCAS focuses upon is if future power market in the midwestern US would be able to support a competitive electricity market when subject to market power. Affiliated with the main research question, conditions under which companies may be in a position to exercise in portions of the study area can be identified. Both research questions are evaluated by checking the evolution of prices and the market allowance for market share competition, thereby also considering potential entrance of new companies to the market.

Generation companies are modelled as agents who constantly explore new strategies in an attempt to exploit the physical limitations of the grid and the market rules in order to always perform better than they have performed in the past. By evaluating past bidding strategies, analysing the competition and anticipating future conditions, a generation company can exhibit learning behaviour and reformulate its bidding strategy subject to environmental factors as forecasted demand, capacity reserve margins and generator risk characteristics.

The Independent System Operator (ISO) or Regional Transmission Operator (RTO) agent is responsible for operating the transmission grid in order to supply power while maintaining safety and reliability. The ISO is not modelled as a strategic agent but rather as an input interface where the user can specify various ISO operating rules - among which are present the required levels of regulation, spinning reserves, non-spinning reserves and replacement reserves - to study the impact of various policy issues on market performance. The user can also specify different price setting rules and settlement rules present in the market. The role of the ISO/RTO is to publish its weather, demand and system available generation capacity forecast to all participants. The ISO/RTO also gathers the bids and offers submitted by the generation and demand companies from which it calculates the market clearing price to optimise the next day generator operations schedule. It is also the responsability of the ISO/RTO to calculate the payments and receipts from the generating companies, demand agents and transmission companies according to the user-specified settlement rules. All other market participants - demand companies, 
transmission companies, distribution companies and regulators - also do not exhibit any strategic behaviour.

Interactions between generation companies and demand companies can occur via forward markets, bilateral contract markets, pool markets and ancillary services markets organised by the ISO. The inclusion of bilateral contracts is the strength of the model. Multi-year bilateral contracts reflect capacity expansion plans and year-ahead marketing strategies to improve performance during the upcoming year. Monthly bilateral contracts between individual demand and generation agents are established based on planned maintenance schedules and to optimise performance over the upcoming year. At last weekly bilateral contracts are considered to adjust according to the generator's short-term optimisation strategy.

Existing and potential future transmission grid configurations are obtained by the NERC in order to apply the model on the electricity market in the mid-western US. The benchmark case implies offering power at production costs and allows relative comparisons between the different scenarios [21] [22].

\section{B. The AMES model}

The Agent-based Modeling of Electricity Systems (AMES) Wholesale Power Market Test bed is an open-source agentbased computational laboratory designed for the systematic study of a centrally administered wholesale power markets operating over AC transmission grids subject to congestion. AMES has been developed to adress the issue of incomplete commercially available packages at the time and attempts to unify researchers, strategic market participants and regulators in order to further develop the model for research, teaching and training purposes. AMES incorporates the elements of the wholesale power market design as recommended by the Federal Energy Regulatory Commission (FERC). This allows a practical model application for many regions in the US ranging from the midwest to the southwest, thereby comprising over $50 \%$ of the generating capacity in the US [23].

Market participants include bulk-power buyers, bulk-power sellers - both distributed along the grid - and an ISO entity. Bulk-power buyers - called Load-Serving Entities (LSE) in the model - aim at securing power for their downstream retail customers by bidding both a linear 24-hour fixed demand bid as an hourly linear price-sensitive demand bid on the day-ahead market, to the ISO. These demand bids are userspecified in the beginning of each simulation run which turn the LSE into a non-strategic entity in the model.

Bulk-power sellers - called generation companies (GenCos) in the model - aim at maximising their daily net earnings by choosing the right linear supply offer to bid on the day-ahead market from their action domain. Sellers are thus modelled as strategic entities capable of learning based on the feedback from the ISO in order to improve their strategies. A generation company can alter its strategy by adjusting the slope of its marginal cost function and by adjusting the upper limit of its reported operating capacity interval.

The ISO guards the operational efficiency of the wholesale power market by operating a day-ahead market and by con- ducting optimal power flow analysis in order to anticipate to transmission grid congestion. The ISO calculates the locational marginal prices on the basis of the GenCos offers and the LSEs bids and publicly reports the hourly commitments to the market participants. At the end of each day the ISO settles commitments on the basis of locational marginal prices and communicates them together with the prices through a public report. This report serves as a basis for the GenCos to use stochastic reinforcement learning in order to adapt their strategy for the next day.

System disturbances such as the volatile electricity production of Renewable Energy Sources (RES-E) or sudden outages fall out of the scope of this model as well as the entry or exit of traders. As a result there is no modelling of real-time market trading. The benchmark case considers the system as if generators would submit supply offers which reflect their true costs [24] [25].

\section{The OPTIMATE Model}

OPTIMATE is an open platform to test integration in new market designs of massive intermittent energy sources dispersed in several regional European power markets. The model targets at evaluating and comparing various existing and potential new market designs aiming at the integration of massive intermittent energy sources dispersed in several regional power markets in Europe. The platform will help developing technical and regulatory solutions compatible with a virtual single European Grid and regional network management processes by assessing, through combined network and market modelling, the expected outputs of new market designs in support of the 2020 EU27 targets. The overarching goal this model strives at is therefore to address intermittent generation capacity issues in a multiple area system by jointly modelling the interactions between market stakeholders and the interarea issues when flow-based market coupling is implemented in order to bridge the gap with current designs.

Intermittent generation has characterisitics that do not fit easily in current electricity market frameworks. Day-ahead wind or solar production forecasts are not reliable with wind energy characterised by a 20 percent day-ahead forecast error relative to the full installed wind capacity. These errors existing at dayahead gate closure does not allow RES-E energy sources to be profitable without the disproportional socialisation of their operating costs through feed-in tariffs.

A promising way to make intermittent generation profitable through the market is the use of flow-based market coupling. A flow-based model reveals which branches in the network physically limit cross-border power exchanges. Although some markets - such as Belgium, France and Germany which together form the Central West European (CWE) region - have already implemented an advanced flow-based market coupling mechanism in order to foster enhanced regional coordination a formal study is needed to back the profitability issue of intermittent generation in Europe.

Market participant behaviour is assumed to be realistic. Agentbased modelling is used to represent strategic market player 
behaviour in order to fully grasp the effect of large-scale intermittent energy source penetration in European electricity markets under the assumption of different market designs. The innovative approach of not focussing on only one market design but keeping the model flexible and open for other existing - and even new market designs - has not been found in existing literature by the author.

\section{CONCLUSION}

Building high quality software for real-world applications is difficult, especially when the modelled system is characterised by flexibility, interactivity and adaptability. Agent-based simulation is a tool which comprises not only the economic, but also the social and environmental factors operating in the system. This makes agent-based simulation a favourable tool for decision makers, especially those who are active in the electric power market. Although any agent-based electric market models have been designed there is still a need for a more flexible model having not only the ability to examine strategic behaviour within a certain market framework, but also to evaluate the performance of the market framework compared with other existing or even new ones. The OPTIMATE model is an up-coming auspicious tool that can fill the gap between the present requests of decision makers and the ability of models existing today.

\section{REFERENCES}

[1] George Gross, "Evolving Nature of Electricity Market Design in the U.S." in Proceedings of the IERE General Meeting and Central \& Eastern European Forum, October 2004.

[2] Kumkar, Lars , "'The California Electricity Market Debacle - On Liberalization Promises and Regulatory Failures"," SSRN eLibrary, 2002.

[3] David Newbery, "Electricity liberalisation in Britain: the quest for a satisfactory wholesale market design," in Cambridge SESSA Conference "Refining Market Design", ser. Cambridge Working Papers in Economics, Cambridge-MIT Institute. Faculty of Economics, University of Cambridge, November 2004

[4] "Electricity market reform failures: UK, Norway, Alberta and California," Energy Policy, vol. 31, no. 11, pp. 1103 - 1115, 2003, Themes in current Asian energy.

[5] N. R. Jennings and K. Sycara and M. Wooldridge, "A Roadmap of Agent Research and Development," Journal of Autonomous Agents and Multi-Agent Systems, vol. 1, no. 1, pp. 7-38, 1998.

[6] Müller,Jörg P., "Architectures and applications of intelligent agents: A survey," The Knowledge Engineering Review, vol. 13, no. 04, pp. 353380, 1999.

[7] Gerhard Weiss, Multiagent systems: a modern approach to distributed artificial intelligence, ed., ser. . Cambridge, MA: The MIT Press, July 2000, vol. ,

[8] Michael Wooldridge, An Introduction to MultiAgent Systems - Second Edition, ed., ser. . : John Wiley \& Sons, May 2009, vol. ,

[9] Christoph Schlueter Langdon, "Agent-Based Modeling for Simulation of Complex Business Systems: Research Design and Validation Strategies," International Journal of Intelligent Information Technologies, Vol. 1, Issue 3, vol. , no. , pp. 1-13, 2005,

[10] Robin B. Matthews and Nigel G. Gilbert and Alan Roach and J. Gary Polhill and Nick M. Gotts, "Agent-based land-use models: a review of applications," Landscape Ecology, vol. 22, no. 10, pp. 1447-1459, December 2007,

[11] "An analysis of agent-based approaches to transport logistics," Transportation Research Part C: Emerging Technologies, vol. 13, no. 4, pp. 255 - 271, 2005, Agents in Traffic and Transportation: Exploring Autonomy in Logistics, Management, Simulation, and Cooperative Driving.

[12] Charles M. Macal and Michael J. North, "Validation of an Agentbased Model of Deregulated Electric Power Markets," in Proc. North
American Computational Social and Organization Science (NAACSOS) 2005 Conference, South, 2005.

[13] H. Van Dyke Parunak, Industrial and Practical Applications of DAI, ed., ser. . Cambridge, MA: The MIT Press, July 1999, vol. In Weiss G. Multiagent Systems: A Modern Approach to Distributed Artificial Intelligence., ch. 9,

[14] Happe, Kathrin, "Agricultural policies and farm structures - Agent-based modelling and application to EU-policy reform," Institute of Agricultural Development in Central and Eastern Europe (IAMO), Studies on the Agricultural and Food Sector in Central and Eastern Europe, 2004.

[15] Happe, Kathrin and Balmann, Alfons and Kellermann, Konrad, "The Agricultural Policy Simulator (Agripolis) An Agent-Based Model To Study Structural Change In Agriculture (Version 1.0)," Institute of Agricultural Development in Central and Eastern Europe (IAMO), IAMO Discussion Papers, 2004.

[16] Werden, G.J. and G.J. and Froeb, L.M., "Simulation as an Alternative to Structural Merger Policy in Differentiated Products Industries," U.S. Department of Justice - Antitrust Division, Papers, 1995.

[17] Wolfe, Shawn R. and Enomoto, Francis Y. and Jarvis, Peter A. and Sierhuis, Maarten, "Comparing Route Selection Strategies in Collaborative Traffic Flow Management," in IAT '07: Proceedings of the 2007 IEEE/WIC/ACM International Conference on Intelligent Agent Technology. Washington, DC, USA: IEEE Computer Society, 2007, pp. 59-62.

[18] Thorsten Schelhorn and David O'Sullivan, "STREETS: an agent-based pedestrian model," Institut fur Umweltsystemforschung Universitat Osnabruck and Centre for Advanced Spatial Analysis University College London, Papers, 1999.

[19] "A multi-agent intelligent system for efficient ERP maintenance," Expert Systems with Applications, vol. 21, no. 4, pp. 191 - 202, 2001, .

[20] "Conceptualizing co-ordination and competition in supply chains as complex adaptive system," Information Systems and E-Business Management, vol. 4, no. 1 , pp. $71-81$, 2006,

[21] Michael North and Guenter Conzelmann and Vladimir Koritarov and Charles Macal and Prakash Thimmapuram and Thomas Veselka, "ELaboratories: Agent-based modeling of Electricity Markets," Center for Energy, Environmental, and Economic Systems Analysis (CEEESA), Papers, 2002.

[22] Guenter Conzelmann and Michael North and Gale Boyd and Richard Cirillo and Vladimir Koritarov and Charles Macal and Prakash Thimmapuram and Tom Veselka, "'Simulating Strategic Market Behavior Using an Agent-Based Modeling Approach: Results of a Power Market Analysis for the Midwestern United States"," in Proceedings of the 6th IAEE European Energy Conference on Modeling in Energy Economics and Policy, September 2004.

[23] Joskow, Paul, "MARKETS FOR POWER IN THE UNITED STATES AN INTERIM ASSESSMENT," Massachusetts Institute of Technology, Center for Energy and Environmental Policy Research, Working Papers, 2005.

[24] Somani, Abhishek and Tesfatsion, Leigh S., "An Agent-Based Test Bed Study of Wholesale Power Market Performance Measures," Iowa State University, Department of Economics, Staff General Research Papers, Aug. 2008.

[25] Li, Hongyan and Tesfatsion, Leigh S., "The AMES Wholesale Power Market Test Bed: A Computational Laboratory for Research, Teaching, and Training," Iowa State University, Department of Economics, Staff General Research Papers, Jun. 2009.

[26] Duan, Rui and Deconinck, Geert, Multi-agent Coordination for Market Environments: Towards a Next Generation Electricity Infrastructure based on Microgrids, ed., ser. Intelligent Systems, Control and Automation: Science and Engineering. : Springer Netherlands, November 2009 , vol. 42 , ch. 7 , 